\title{
High SARS-CoV-2 Seroprevalence in Rural Peru, 2021: a Cross-Sectional Population-Based Study
}

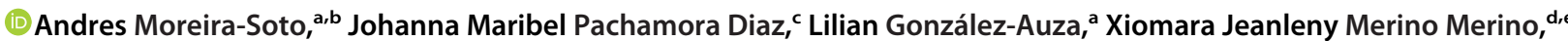 \\ Alvaro Schwalb, ${ }^{\text {d,e }}$ Christian Drosten, ${ }^{a}$ Eduardo Gotuzzo, ${ }^{\text {d,e }}$ Michael Talledo, ${ }^{\text {d,e }}$ Heriberto Arévalo Ramirez, ${ }^{c}$ Roxana Peralta Delgado, ${ }^{c}$ \\ Spassky Bocanegra Vargas, ${ }^{\mathrm{f}}$ J Jan Felix Drexler ${ }^{\mathrm{a}, \mathrm{g}}$ \\ aCharité-Universitätsmedizin Berlin, corporate member of Freie Universität Berlin and Humboldt-Universität zu Berlin, Institute of Virology, Berlin, Germany \\ bVirology-CIET, Faculty of Microbiology, University of Costa Rica, San José, Costa Rica \\ cDirección Regional de Salud de San Martín, Moyobamba, Peru \\ alnstituto de Medicina Tropical Alexander von Humboldt, Universidad Peruana Cayetano Heredia, Lima, Peru \\ eDepartamento de Medicina, Facultad de Medicina, Universidad Peruana Cayetano Heredia, Lima, Peru \\ IMoyobamba Hospital, Moyobamba, Peru \\ פGerman Centre for Infection Research (DZIF), associated partner site Charité, Berlin, Germany
}

ABSTRACT Latin America has been severely affected by the COVID-19 pandemic. The COVID-19 burden in rural settings in Latin America is unclear. We performed a cross-sectional, population-based, random-selection SARS-CoV-2 serologic study during March 2021 in the rural population of San Martin region, northern Peru. In total, 563 persons from 288 houses across 10 provinces were enrolled, reaching $0.2 \%$ of the total rural population of San Martin. Screening for SARS-CoV-2 IgG antibodies was done using a chemiluminescence immunoassay (CLIA), and reactive sera were confirmed using a SARS-CoV-2 surrogate virus neutralization test (sVNT). Validation of the testing algorithm using prepandemic sera from two regions of Peru showed false-positive results in the CLIA (23/84 sera; $27 \%$ ) but not in the sVNT, highlighting the pitfalls of SARS-CoV-2 antibody testing in tropical regions and the high specificity of the two-step algorithm used in this study. An overall 59.0\% seroprevalence $(95 \%$ confidence interval $[\mathrm{Cl}, 55$ to 63\%) corroborated intense SARS-CoV-2 spread in San Martin. Seroprevalence rates between the 10 provinces varied from 41.3 to $74.0 \%$ (95\% Cl, 30 to $84 \%)$. Higher seroprevalence was not associated with population size, population density, surface area, mean altitude, or poverty index in Spearman correlations. Seroprevalence and reported incidence diverged substantially between provinces, suggesting regional biases of COVID-19 surveillance data. Potentially, limited health care access due to environmental, economic, and cultural factors might lead to undetected infections in rural populations. Additionally, test avoidance to evade mandatory quarantine might affect rural regions more than urban regions. Serologic diagnostics should be pursued in resource-limited settings to inform country-level surveillance and vaccination strategies and to support control measures for COVID-19.

IMPORTANCE Latin America is a global hot spot of the COVID-19 pandemic. Serologic studies in Latin America have been mostly performed in urban settings. Rural populations comprise $20 \%$ of the total Latin American population. Nevertheless, information on COVID-19 spread in rural settings is scarce. Using a representative population-based seroprevalence study, we detected a high seroprevalence in rural populations in San Martin, northern Peru, in 2021, reaching 41 to $74 \%$. However, seroprevalence and reported incidence diverged substantially between regions, potentially due to limited health care access or test avoidance due to mandatory quarantine. Our results suggest that rural populations are highly affected by SARS-CoV-2 even though they are sociodemographically distinct from urban populations and that highly specific serological
Editor Seema Lakdawala, University of Pittsburgh School of Medicine Copyright $\odot 2021$ Moreira-Soto et al. This is an open-access article distributed under the terms of the Creative Commons Attribution 4.0 International license. Address correspondence to Jan Felix Drexler, felix.drexler@charite.de.

Received 13 August 2021

Accepted 29 October 2021

Published 24 November 2021 
diagnostics should be performed in resource-limited settings to support public health strategies of COVID-19 control.

KEYWORDS SARS-CoV-2, Peru, COVID-19, serology, seroprevalence, rural, Latin America

eru has been severely affected by the COVID-19 pandemic, with one of the highest mortality per capita reported worldwide since the start of the pandemic, reaching 6,132 deaths per million as of October $2021(1,2)$. The determinants of SARS-CoV-2 spread in Latin America are poorly defined. An epidemiological study from European and Asian urban centers correlated population density with increased SARS-CoV-2 infection rates (3). In contrast, epidemiological studies from Latin American urban centers yielded diverse prevalence estimates that seemed uncorrelated with population density, exemplified by the three available seroprevalence studies from Peru, conducted during mid- to late 2020. The first study from the Peruvian capital, Lima (popu-

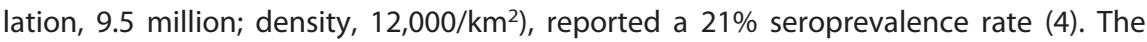
second study from the Lambayeque department in northern Peru (population, 1.2 million; density, $84 / \mathrm{km}^{2}$ ) reported a $29 \%$ seroprevalence rate (5). The third study from Iquitos city, Peruvian Amazon (population, 470,000; density, 417/ $\mathrm{km}^{2}$ ), reported a $70 \%$ seroprevalence rate, implying local herd immunity had been reached (6). Despite the high seroprevalence already during 2020, a second SARS-CoV-2 wave occurred in Iquitos in January 2021. Potentially, mobile susceptible populations in the Amazon region might explain the occurrence of a second wave in lquitos (6). Susceptible populations might correspond to rural and indigenous populations, comprising up to $19 \%$ of the total Latin American population based on World Bank estimates (https://data .worldbank.org/indicator/SP.RUR.TOTL.ZS?locations=ZJ). The high geographical dispersion and low population density of these communities, in addition to regular traveling to urban centers for commerce, might contribute to a differential dispersion of SARSCoV-2 compared to urban settings.

Seroprevalence study in San Martin. Our study focused on San Martin, Peru (total population, 899,648; density, $\left.18 / \mathrm{km}^{2}\right)$. The San Martin department $\left(51,000 \mathrm{~km}^{2}\right)$ is located in the north of Peru (Fig. 1A). The department encompasses diverse ecosystems such as valleys, Andes mountains, and Amazon rainforests, reaching altitudes from 190 to $3,080 \mathrm{~m}$ (Fig. 1A), and comprises $\leq 5 \%$ of Peruvian forest cover. The San Martin population is divided into Mestizo (83.6\%) followed by Quechua (5.3\%) and Afro-Peruvian (4.9\%) ethnicities per census data of the National Institute of Statistics and Census of Peru (INEl; www.inei.gob.pe/).

We performed a cross-sectional population-based random-selection study during March 2021 (institutional ethics committee Via Libre; number 6532-2021a) in the 10 provinces of San Martin (Fig. 1B). Sample size calculation considered the rural population of San Martin (286,988), a 95\% confidence level, and an estimated SARS-CoV-2 seroprevalence of $50 \%$, reaching 576 individuals. As the average household in Peru consists of 4 inhabitants (www.inei.gob.pe/), the study aimed at sampling two individuals from 288 individual houses. First, a conglomerate of 100 rural houses was selected based on estimates of the census data of 2017 and on geographic information systems of the INEI. Next, a random household selection in the conglomerate was performed. Exclusion criteria encompassed populations residing in collective dwellings such as barracks, police stations, convents, boarding schools, and hotels, used as temporary housing of unrelated people with unstable population dynamics; age $\leq 5$ years; skin lesions in the venous puncture site; use of alcohol or psychoactive drugs; not being a permanent resident; and not signing the informed consent form. Participation was voluntary, and participant selection in households was performed using a Kish grid (7). A $10-\mathrm{ml}$ blood sample was taken after the informed consent was signed by the person or the caretaker if the person was $\leq 18$ years of age. We obtained samples from 563 persons visiting 288 houses in the 10 provinces, comprising $0.2 \%$ of the total rural population of San Martin. The sampled cohort included persons aged 6 to 89 years (mean, 
A

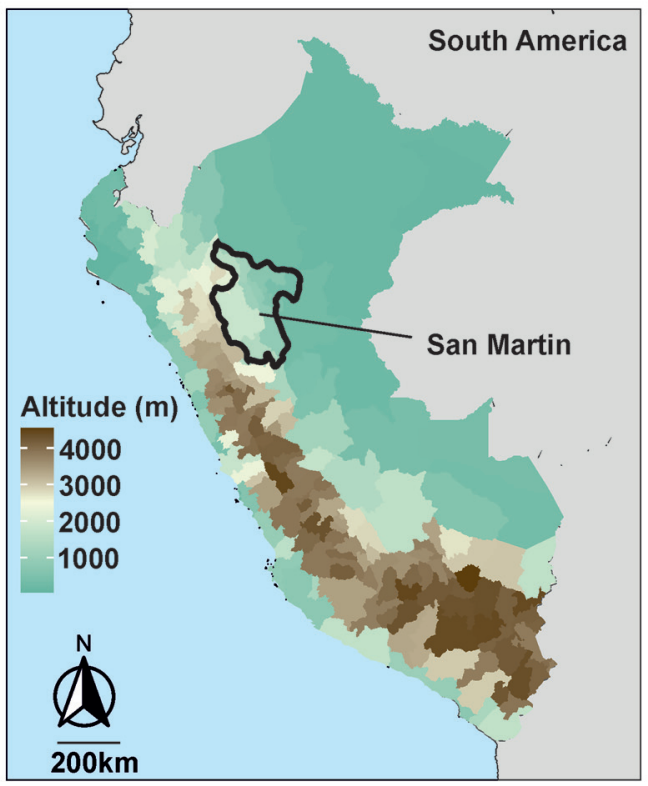

C SARS-CoV-2 seroprevalence study from San Martin, Peru, 2021

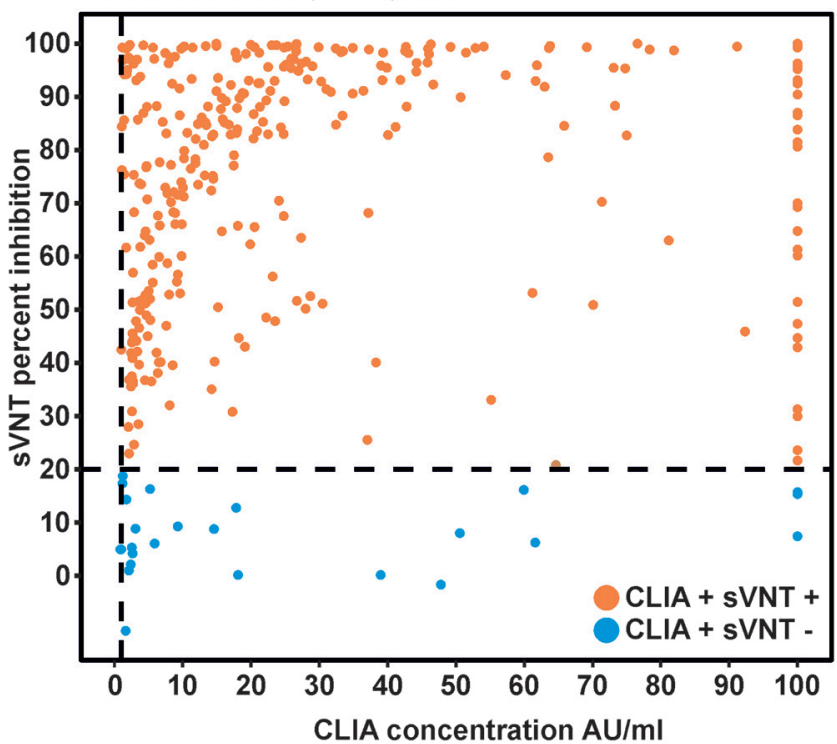

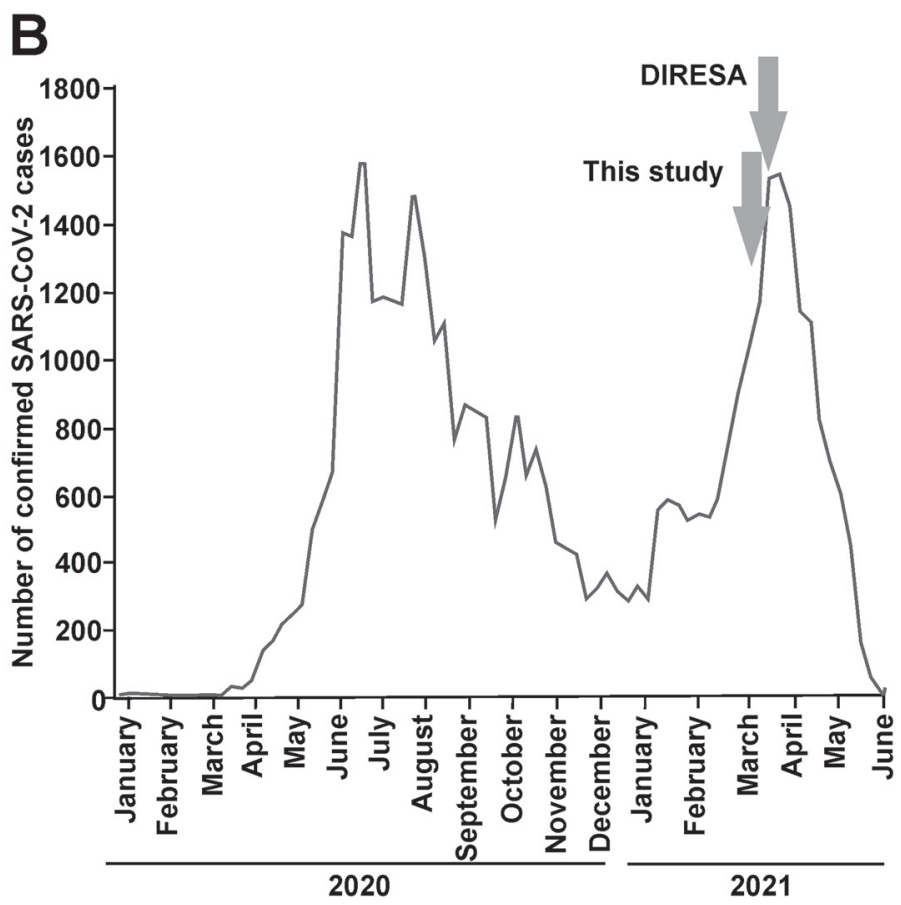

D Pre-COVID-19 samples from Cuzco and Loreto, Peru, 2013-2018



FIG 1 Epidemiological surveillance and serologic diagnostics of SARS-CoV-2 from San Martin, Peru. (A) Mean altitude (meters above sea level) map of Peru. The department of San Martin is circled in black. (B) Number of confirmed cases in San Martin as of June 2021. The times where incidence data and samples for the serologic study were taken are marked with arrows. Surveillance data were gathered from https://diressanmartin.gob.pe/ and https://www.gob.pe/minsa/. Reactivity of serum samples from SARS-CoV-2 seroprevalence study in San Martin in 2021 (C) and pre-COVID-19 samples from 2013 to 2018 from Peru (D) in a chemiluminescence immunoassay (CLIA), shown in the $x$ axis, and a SARS-CoV-2 surrogate virus neutralization test (sVNT), shown in the $y$ axis. AU/ml, absorbance unit per milliliter.

35.8; standard deviation [SD]: 21.15) consistent with San Martin's age distribution data from the INEI 2017 census (see Fig. S1 in the supplemental material; www.inei.gob.pe/). From the cohort, $37.7 \%$ (212/563) were male and 62.3\% (351/563) female, contrasting with the sex distribution in San Martin (51.3\% male, $48.7 \%$ female). The difference between the sex distribution can be explained by the sampling strategy, limited to visiting the homes of the participants during the day due to safety and operational reasons, while males were mostly out working.

Two-step serologic testing and validation. Using a chemiluminescence immunoassay (CLIA) (SARS-CoV-2 S-RBD IgG kit; Snibe Diagnostic, China), we detected SARS- 
CoV-2-specific IgG antibodies in $63.6 \%$ of the samples (358/563) (Fig. 1C and Fig. S2). To validate the CLIA results, we used 84 pre-COVID-19 samples from our previous study of arbovirus serology in Peru (8) (Fig. 1D and Fig. S2). The samples were collected in the departments of Cuzco and Loreto during 2013 and 2018, respectively. A total of 27\% (23/84; 11 from Cuzco and 12 from Loreto) of pre-COVID-19 samples yielded positive results in the CLIA irrespective of geographical location (Fig. 1D and Fig. S2). The apparently positive pre-COVID-19 samples showed statistically significantly lower signal concentrations in the CLIA than those samples from 2021 (median pre-COVID$19=1.6$ versus COVID-19 $=32.81 ; t$ test, $P<0.001$ ) (Fig. S2), suggesting unspecific reactivity. Unspecific reactivity may be elicited by antibodies against common-cold coronaviruses (9) or endemic tropical diseases such as malaria, dengue, and Zika, either by polylconal B-cell stimulation or by weakly cross-reactive antibodies $(10,11)$. Therefore, a confirmatory SARS-CoV-2 surrogate virus neutralization test (sVNT; GenScript, USA) was performed in all CLIA-reactive samples. A total of 92.7\% (332/358) of the 2021 CLIA-positive samples were confirmed using the sVNT, whereby minor differences between CLIA and sVNT results can be attributed to differential sensitivity of the tests. (Fig. 1C and Fig. S2). In stark contrast, none of the CLIA-positive prepandemic samples yielded positive results in the sVNT. These results corroborate that unspecific reactivity of serologic tests in tropical areas must be carefully evaluated (10) and highlight the robustness of our serologic testing algorithm. Therefore, only samples yielding positive results in both serologic assays were considered for further analyses in our study to maximize specificity.

Seroprevalence and statistical analyses. Overall, the SARS-CoV-2 seroprevalence for San Martin was 59.0\% (332/563; 95\% confidence interval [CI], 55 to 63\%). No statistically significant difference of seroprevalence per sex was observed, using a chi-square test ( $\chi^{2}=0.05 ; P=0.83$ ). However, a statistically significant difference was observed between the ages of SARS-CoV-2-seropositive and SARS-CoV-2-seronegative persons using a $t$ test ( 33 years [range, 6 to 89 ] versus 38 years [range, 7 to $57 ; P=0.01$ ]). This observation was in concordance with the previously mentioned study from lquitos, likely associated with higher contact rates facilitating SARS-CoV-2 transmission in younger age groups (6).

Seroprevalence between the 10 provinces varied from 41.3 to $74.0 \%(\mathrm{Cl}, 30$ to $84 \%)$ (Fig. 2A and B and Table S1). Higher seroprevalence was neither associated with population size (Spearman correlation test, $\left.r_{s}=-0.26 ; P=0.46\right)$, population density $\left(r_{s}=\right.$ $-0.14 ; P=0.68)$, surface area $\left(r_{s}=0.33 ; P=0.34\right)$, mean altitude of the province $\left(r_{s}=\right.$ -0.59; $P=0.06)$, nor poverty index $\left(r_{s}=0.10 ; P=0.76\right)$ (Fig. $2 C$ and Fig. S3). Interestingly, the cumulative epidemiological surveillance data from the Regional Health Directorate of Peru (DIRESA) relying on notified cases of acute SARS-CoV-2 infection confirmed by either reverse transcription-PCR (RT-PCR) or an antigen test (Fig. 2B) showed no statistically significant correlation with our serologic data although cumulatively measuring similar time spans, encompassing early 2020 to March $2021\left(r_{s}=\right.$ $-0.14 ; P=0.68$ ) (Fig. 1B and Fig. 2D). On the one hand, overall higher seroprevalence than reported cases is consistent with high numbers of undetected asymptomatic COVID-19 cases (12). On the other hand, we detected two provinces with a relatively higher divergence of seroprevalence compared to incidence data (Mariscal Caceres and Bellavista, Fig. 2A, B, and D). Hypothetically, individuals in those provinces may lack health care access due to numerous environmental, economic, and cultural factors (13), which might lead to more undetected infections in these rural populations. First, test refusal to avoid mandatory quarantine or stigmatization has been reported for SARS-CoV2 from different countries $(14,15)$. Test avoidance might be greater in rural regions of resource-limited areas, where a high share of the population work under conditions that are not compatible with quarantine and hospital visits. Second, local health centers might be difficult to access or not be sufficiently equipped for the high COVID-19 burden (16), limiting access to diagnostics in rural areas. Third, high infection rates in rural populations might be explained by lack of access to basic housing elements such as 
A

SARS-CoV-2 seroprevalence from this study, March 2021

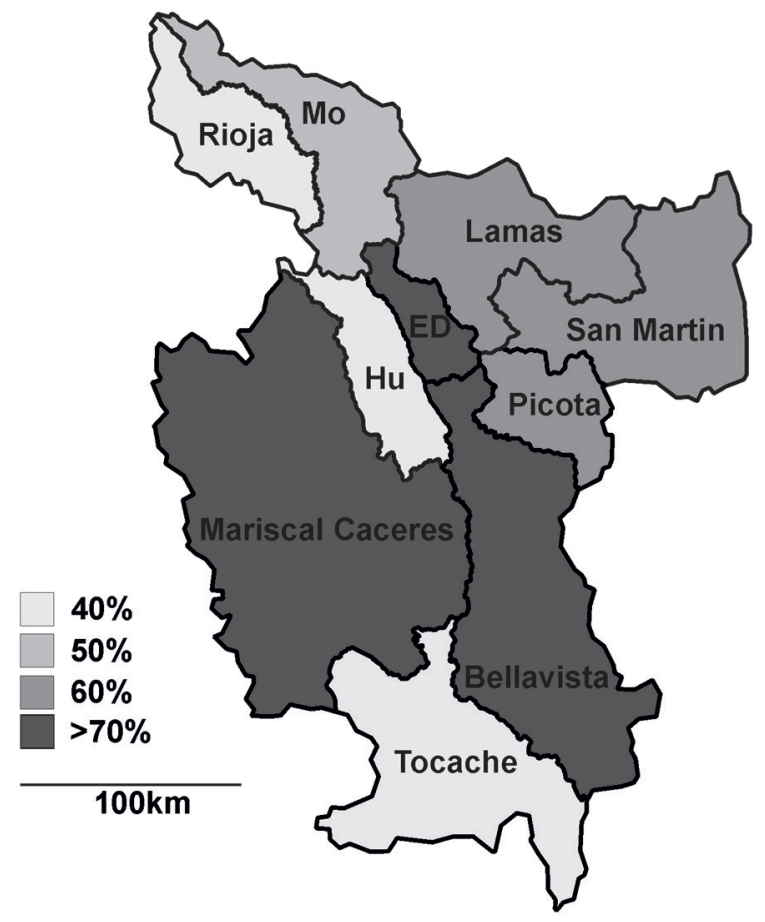

C

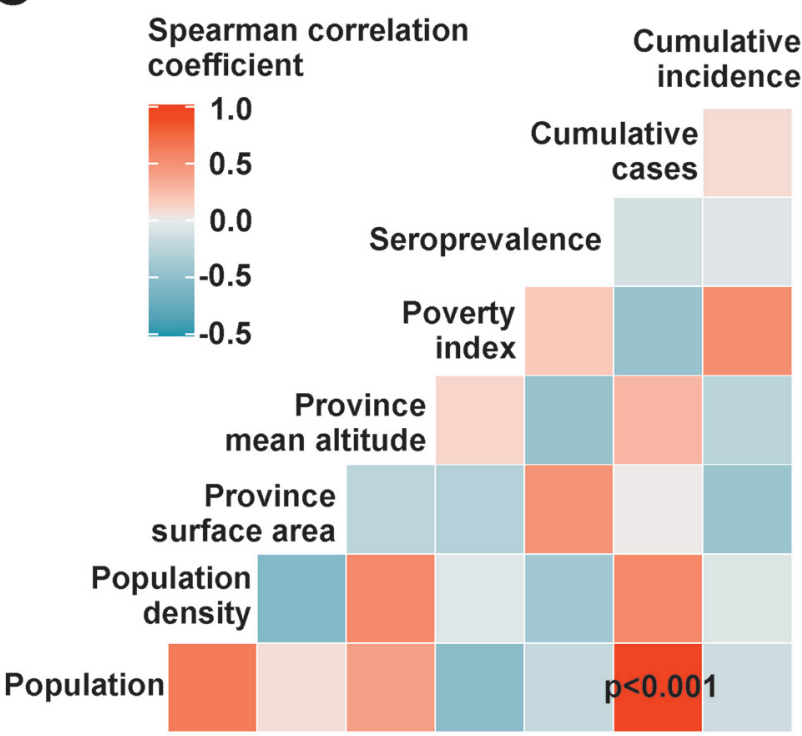

B DIRESA cumulative incidence, March 13th, 2021

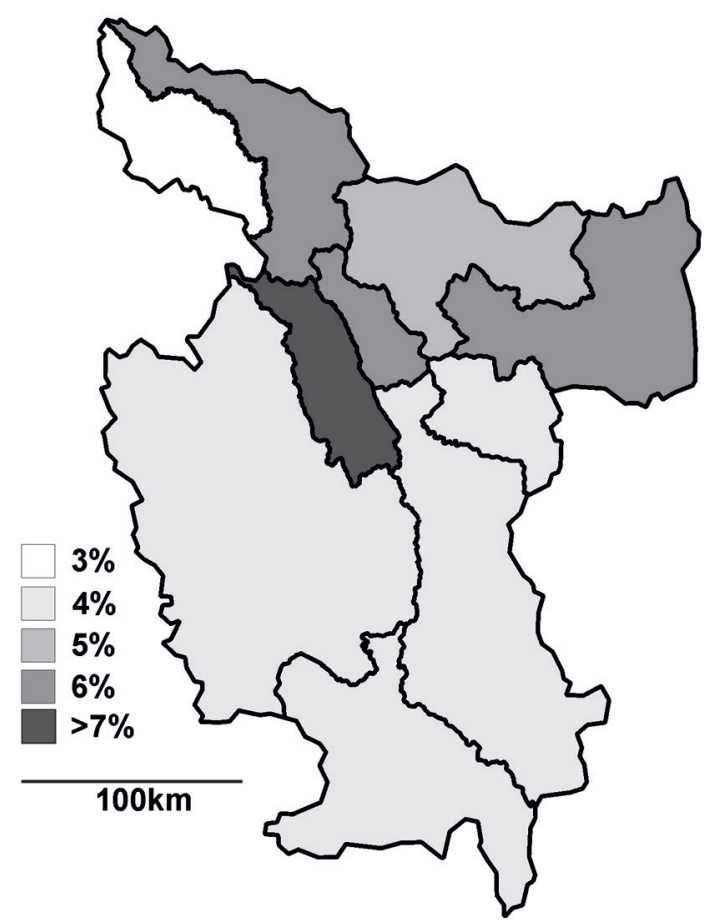

D

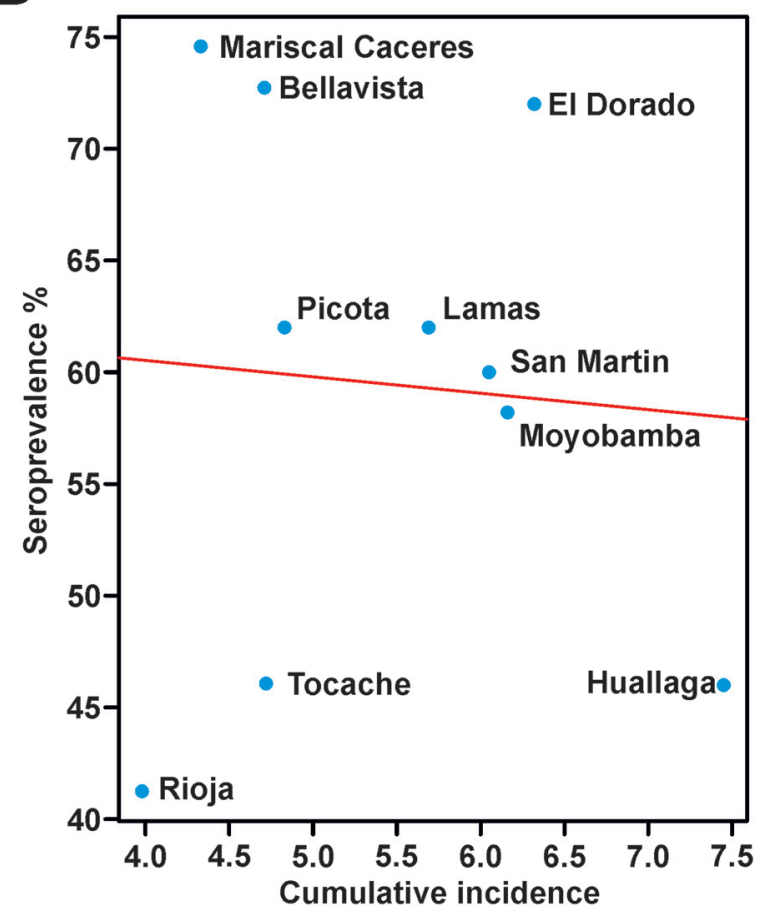

FIG 2 Correlation of seroprevalence and incidence data with different indicators. (A and B) Comparison of serologic (A) and incidence (B) data from San Martin. Hu, Huallaga; Mo, Moyobamba; ED, El Dorado. (C) Heatmap of Spearman's rank correlation test using different social, economic, and geographical indicators. Significant correlations are depicted inside the square. (D) Spearman's rank correlation test of seroprevalence and cumulative incidence per province.

sewage disposal systems, as observed in rural populations in Ecuador (17). Similarly, a survey analyzing rural residents in the United States found that they were less likely to participate in COVID-19 preventive measures such as the use of masks in public or working from home, increasing the possibility of infection (18). Another province 
(Huallaga, Fig. 2A, B, and D) showed higher cumulative incidence than other provinces for unknown reasons, substantiating inconsistencies between serologic and incidence data.

Irrespective of the underlying reasons leading to the overall high seroprevalence, our data suggest a large number of undiagnosed COVID-19 cases potentially challenging test-trace-isolate interventions in the region (19). Previous studies in the United States have stressed that rural regions are particularly vulnerable to COVID-19, leading to higher mortality rates in rural than in urban regions, and with higher mortality rates associated with black and Hispanic populations $(20,21)$, suggesting further studies are needed in Latin American vulnerable populations. Limitations of our study include limited metadata and potential sampling biases. However, thorough study design, exhaustive serologic testing, and high seroprevalence consistent with data from other Latin American settings suggest robustness of our results $(6,17)$. Serologic diagnostics should be pursued in resource-limited settings to inform country-level surveillance and vaccination strategies and to support control measures for COVID-19.

\section{SUPPLEMENTAL MATERIAL}

Supplemental material is available online only.

FIG S1, TIF file, 1.8 MB.

FIG S2, TIF file, 1.8 MB.

FIG S3, TIF file, 1.8 MB.

TABLE S1, DOCX file, $0.02 \mathrm{MB}$.

\section{ACKNOWLEDGMENTS}

We thank the DIRESA workers who aided in the sample collection and all study

participants. We thank Victor Carvalho for technical support.

We report no conflicts of interest.

This work was supported by the Deutsche Gesellschaft für Internationale Zusammenarbeit (GIZ) GmbH contract number 88114104.

\section{REFERENCES}

1. Munayco C, Chowell G, Tariq A, Undurraga EA, Mizumoto K. 2020. Risk of death by age and gender from CoVID-19 in Peru, March-May, 2020. Aging (Albany NY) 12:13869-13881. https://doi.org/10.18632/aging.103687.

2. Roser M. 2020. Coronavirus pandemic (COVID-19). Our World in Data. https://ourworldindata.org/coronavirus.

3. Diao Y, Kodera S, Anzai D, Gomez-Tames J, Rashed EA, Hirata A. 2021. Influence of population density, temperature, and absolute humidity on spread and decay durations of COVID-19: a comparative study of scenarios in China, England, Germany, and Japan. One Health 12:100203. https://doi.org/10.1016/j.onehlt.2020.100203.

4. Reyes-Vega MF, Soto-Cabezas MG, Cárdenas F, Martel KS, Valle A, Valverde J, Vidal-Anzardo M, Falcón ME, Munayco CV, Peru COVID-19 Working Group. 2021. SARS-CoV-2 prevalence associated to low socioeconomic status and overcrowding in an LMIC megacity: a population-based seroepidemiological survey in Lima, Peru. EClinicalMedicine 34:100801. https://doi.org/10 .1016/j.eclinm.2021.100801.

5. Díaz-Vélez C, Failoc-Rojas VE, Valladares-Garrido MJ, Colchado J, CarreraAcosta L, Becerra M, Moreno Paico D, Ocampo-Salazar ET. 2021. SARSCoV-2 seroprevalence study in Lambayeque, Peru, June-July 2020. PeerJ 9:e11210. https://doi.org/10.7717/peerj.11210.

6. Alvarez-Antonio C, Meza-Sanchez G, Calampa C, Casanova W, Carey C, Alava F, Rodriguez-Ferrucci H, Quispe AM. 2021. Seroprevalence of antiSARS-CoV-2 antibodies in lquitos, Peru in July and August, 2020: a population-based study. Lancet Glob Health 9:e925-e931. https://doi.org/10 .1016/S2214-109X(21)00173-X.

7. Kish L. 1949. A procedure for objective respondent selection within the household. J Am Stat Assoc 44:380-387. https://doi.org/10.1080/01621459 1949.10483314.

8. Fischer C, Bozza F, Merino Merino XJ, Pedroso C, De Oliveira Filho EF, Moreira-Soto A, Schwalb A, De Lamballerie X, Netto EM, Bozza PT, Sarno M, Brites C, Gotuzzo E, Talledo M, Drexler JF. 2020. Robustness of serologic investigations for Chikungunya and Mayaro viruses following coemergence. mSphere 5:e00915-19. https://doi.org/10.1128/mSphere .00915-19.

9. Hicks J, Klumpp-Thomas $C$, Kalish $\mathrm{H}$, Shunmugavel A, Mehalko J, Denson J-P, Snead KR, Drew M, Corbett KS, Graham BS, Hall MD, Memoli MJ, Esposito D, Sadtler K. 2021. Serologic cross-reactivity of SARS-CoV-2 with endemic and seasonal betacoronaviruses. J Clin Immunol 41:906-913. https://doi.org/10.1007/s10875-021-00997-6.

10. Yadouleton A, Sander AL, Moreira-Soto A, Tchibozo C, Hounkanrin G, Badou Y, Fischer C, Krause N, Akogbeto P, de Oliveira Filho EF, Dossou A, Brunink S, Aissi MAJ, Djingarey MH, Hounkpatin B, Nagel M, Drexler JF. 2021. Limited specificity of serologic tests for SARS-CoV-2 antibody detection, Benin. Emerg Infect Dis 27:233-237. https://doi.org/10.3201/ eid2701.203281.

11. Lustig Y, Keler S, Kolodny R, Ben-Tal N, Atias-Varon D, Shlush E, Gerlic M, Munitz A, Doolman R, Asraf K, Shlush LI, Vivante A. 2021. Potential antigenic cross-reactivity between severe acute respiratory syndrome coronavirus 2 (SARS-CoV-2) and dengue viruses. Clin Infect Dis 73:e2444-e2449. https://doi.org/10.1093/cid/ciaa1207.

12. McArthur L, Sakthivel D, Ataide R, Chan F, Richards JS, Narh CA. 2020. Review of burden, clinical definitions, and management of COVID-19 cases. Am J Trop Med Hyg 103:625-638. https://doi.org/10.4269/ajtmh.20-0564.

13. Peters DJ. 2020. Community susceptibility and resiliency to COVID-19 across the rural-urban continuum in the United States. J Rural Health 36: 446-456. https://doi.org/10.1111/jrh.12477.

14. Rubin R. 2020. First it was masks; now some refuse testing for SARS-CoV2. JAMA 324:2015-2016. https://doi.org/10.1001/jama.2020.22003.

15. McDermott JH, Newman WG. 2020. Refusal of viral testing during the SARS-CoV-2 pandemic. Clin Med (Lond) 20:e163-e164. https://doi.org/10 .7861/clinmed.2020-0388. 
16. Sander A-L, Yadouleton A, Moreira-Soto A, Tchibozo C, Hounkanrin G, Badou Y, Fischer C, Krause N, Akogbeto P, de Oliveira Filho EF, Dossou A, Brünink S, Drosten C, Aïssi MAJ, Harouna Djingarey M, Hounkpatin B, Nagel M, Drexler JF. 2021. An observational laboratory-based assessment of SARS-CoV-2 molecular diagnostics in Benin, Western Africa. mSphere 6:e00979-20. https://doi.org/10.1128/mSphere.00979-20.

17. Del Brutto OH, Costa AF, Mera RM, Recalde BY, Bustos JA, Garcia HH. 2021 SARS-CoV-2 in rural Latin America. A population-based study in coastal Ecuador. Clin Infect Dis 73:314-317. https://doi.org/10.1093/cid/ciaa1055.

18. Callaghan T, Lueck JA, Trujillo KL, Ferdinand AO. 2021. Rural and urban differences in COVID-19 prevention behaviors. J Rural Health 37:287-295. https://doi.org/10.1111/jrh.12556.
19. Contreras S, Dehning J, Loidolt $M$, Zierenberg J, Spitzner FP, Urrea-Quintero JH, Mohr SB, Wilczek M, Wibral M, Priesemann V. 2021. The challenges of containing SARS-CoV-2 via test-traceand-isolate. Nat Commun 12:378. https://doi.org/10.1038/s41467 -020-20699-8.

20. Cheng KJG, Sun Y, Monnat SM. 2020. COVID-19 death rates are higher in rural counties with larger shares of Blacks and Hispanics. J Rural Health 36:602-608. https://doi.org/10.1111/jrh.12511.

21. Huang $Q$, Jackson S, Derakhshan S, Lee L, Pham E, Jackson A, Cutter SL. 2021. Urban-rural differences in COVID-19 exposures and outcomes in the South: a preliminary analysis of South Carolina. PLoS One 16: e0246548. https://doi.org/10.1371/journal.pone.0246548. 